\title{
DOADOR DOMINÓ DE CORAÇÃO: POSSIBILIDADE DE APROVEITAMENTO DAS VALVAS CARDÍACAS
}

\author{
Domino donor heart: possibility of using cardiac valves
}

\author{
César Augusto Guimarães Marcelino, Carine Cristiane Fusco, Mara Nogueira Araújo e Andréa Cotait Ayoub
}

\begin{abstract}
RESUMO
Introdução: As valvopatias destacam-se dentre as cardiopatias cirúrgicas, principalmente em nosso meio, sendo que muitas vezes a conservação da valva é difícil pelo extenso acometimento e a sua substituição torna-se imperativa. Entre os substitutos valvares, as próteses e biopróteses, os substitutos homólogos apresentam vantagens especiais; porém, seu maior fator limitante é a dificuldade de obtenção. Diante dessa situação, os corações nativos explantados nos transplantes podem representar importante fonte de oferta dos substitutos valvares. Objetivo: Verificar a real possibilidade de aproveitamento das valvas cardíacas oriundas de corações nativos explantados de transplante cardíaco. Método: Estudo do tipo exploratório, descritivo e documental, realizado em hospital da rede estadual de São Paulo, referente ao atendimento a doenças cardiovasculares. Resultados: A amostra foi composta por 202 corações de pacientes submetidos a transplante, sendo $155(76,7 \%)$ homens e 47 deles com idade entre 56-70 anos (31,1\%). Em relação à etiologia da cardiomiopatia, encontravam-se assim distribuídas: $51(25,2 \%)$ possuíam tal informação, e destes, a mais frequente foi cardiomiopatia dilatada em 16 casos (31,4\%). Dos 202 corações analisados, $114(56,4 \%)$ a avaliação foi possível, sendo que destes, $69(59,6 \%)$ laudos relataram que as valvas não possuíam alteração, enquanto $46(40,4 \%)$ informavam presença de alteração valvar. As valvas cardíacas com menos frequência de alterações foram as pulmonares, em $82(71,9 \%)$ casos e aórtica em 81 (71,1\%). Conclusão: O estudo anatomopatológico de corações retirados de receptores cardíacos mostrou a existência de valvas cardíacas que poderiam ser utilizadas em transplante valvar, em especial da valva aórtica.
\end{abstract}

Descritores: Valvas Cardíacas; Implante de Prótese de Valva Cardíaca; Doadores de Tecidos; Transplante de Coração; Transplante de Tecidos.

\section{Instituição:}

Organização de Procura de Órgãos, Instituto Dante Pazzanese de Cardiologia, São Paulo, SP

\section{Aspecto Ético}

0 projeto foi aprovado pelo Comitê de Ética em Pesquisa do Instituto Dante Pazzanese de Cardiologia com número de protocolo 3615 de 2008.

\section{Correspondência:}

César Augusto Guimarães Marcelino

Av. Dr. Dante Pazzanese, 500, Prédio II, $3^{\circ}$ andar - CEP: 04012-909, São Paulo-SP, Brasil.

Tel.: (11) 5571-8601 (comercial).

E-mail: cesaraugustomarcelino@bol.com.br, cesarg@dantepazzanese.org.br

\section{INTRODUÇÃO}

O transplante de órgãos e tecidos é um dos avanços mais audaciosos e revolucionários da Medicina. Superando dificuldades e gerando conhecimentos que impulsionaram a ciência médica, os transplantes popularizaram-se e diferentes órgãos e tecidos atualmente podem ser transplantados., ${ }^{1,2}$

O primeiro transplante cardíaco humano foi realizado por Barnard em 1967, na África do Sul. No Brasil, em 1968, Zerbini realizou o primeiro transplante da América Latina e o paciente faleceu de infecção 28 dias após a operação, até que na década de 1980, com a introdução da ciclosporina $\mathrm{A}$, os episódios de rejeição passaram a ter comportamento benigno e de mais fácil controle.

O transplante cardíaco é o tratamento de escolha para insuficiência cardíaca refratária a tratamento clínico ou quando não comporta outras opções cirúrgicas, exigindo-se criteriosa seleção na escolha de doador e receptor. ${ }^{2-5}$ 
O Brasil cada vez mais tem ocupado espaço no campo dos transplantes, destacando-se na América Latina e, sobretudo, sendo país referência no transplante cardíaco na doença de Chagas. ${ }^{4}$

O Instituto Dante Pazzanese de Cardiologia (IDPC) é uma instituição do Estado de São Paulo especializada na assistência a pacientes portadores de doenças cardiovasculares. Realiza transplantes cardíacos, renais e hepáticos. Nele, o primeiro transplante cardíaco ocorreu em 1991 e, desde então, tornou-se um dos centros de referência no Brasil.

Dentro das diversas afecções cardíacas, destaca-se o mal funcionamento das valvas cardíacas humanas, sendo necessária sua substituição por valvas biológicas, metálicas, ou por meio do transplante de valvas humanas procedentes de doador falecido ou de doador dominó.

O conceito "doador dominó de coração" refere-se à modalidade de transplante onde são utilizadas as valvas ou o próprio coração nativo após análise anatomopatológica das estruturas doadas e com consentimento prévio ao transplante cardíaco.

As valvas cardíacas humanas são utilizadas como alternativa substitutiva nas disfunções valvares desde 1962, com vantagens quando comparadas às próteses convencionais, devido ao melhor desempenho hemodinâmico, baixa incidência de tromboembolismo, menores índices de infecções e não necessidade de anticoagulação. Tais vantagens resultam em melhor qualidade de vida no pósoperatório, e, algumas vezes, maior sobrevida tardia..$^{4,5}$

A durabilidade dos enxertos de valvas pulmonares e o desempenho hemodinâmico estão relatados na literatura, sobretudo quando usados para corrigir defeitos congênitos e na estenose de via de saída de ventrículo direito. ${ }^{6,7}$

As valvas possíveis de transplante são aquelas oriundas de doadores com no máximo 65 anos para valvas aórticas e 60 anos para valvas pulmonares, sendo que as mais processadas são as valvas aórticas, seguida das pulmonares e mitrais..$^{89}$

O transplante de valvas cardíacas humanas segue o mesmo o processo de doação de múltiplos órgãos e tecidos, respeitando o estabelecido pela Lei n ${ }^{\circ} 9434$ de fevereiro de 1997, que dispõe sobre a remoção de órgãos, tecidos e partes do corpo humano para fins de transplante e tratamento. ${ }^{10}$

O primeiro banco de valvas cardíacas humanas no Brasil a ser cadastrado pelo Ministério da Saúde (para captação, processamento e distribuição de enxertos cardiovasculares) surgiu em 1996 no Hospital de Caridade da Irmandade da Santa Casa de Misericórdia de Curitiba. ${ }^{6,8}$

No Estado de São Paulo não há banco de enxertos valvares, o que impossibilita o processamento, estocagem e distribuição desse tecido.

\section{OBJETIVO}

Verificar a possibilidade de aproveitamento do tecido valvar presente nos corações explantados de pacientes submetidos a transplante cardíaco no período de 1991 a 2007 e inferir a taxa de utilização das valvas obtidas.

\section{MÉTODO}

O estudo foi do tipo exploratório, descritivo, documental e retrospectivo realizado em uma Organização de Procura de Órgãos (OPO) de uma instituição pública da cidade de São Paulo referência em doenças cardiovasculares, considerada também uma instituição captadora e transplantadora cardíaca, renal e hepática.

Foram consultados os laudos dos exames anatomopatológicos de corações explantados de pacientes submetidos a transplante cardíaco no período de janeiro de 1991 a janeiro de 2007, tendo como variáveis de estudo: idade, sexo, diagnóstico da cardiomiopatia e alterações morfológicas das valvas (tricúspide, mitral, pulmonar e aórtica).

A análise dos dados foi realizada com o auxílio do estatístico do Laboratório de Epidemiologia e Estatística (LEE) do IDPC. Inicialmente, foi feita uma análise descritiva dos dados, com o objetivo de caracterizar a amostra quanto às variáveis: sexo, idade, diagnóstico e alterações morfológicas das valvas cardíacas (tricúspide, mitral, pulmonar e aórtica). Para comparação múltipla entre sexo, idade, diagnóstico e alterações morfológicas das valvas cardíacas foi utilizado o teste do Qui-quadrado e o teste exato de Fisher, para avaliar se havia associação ou não dos dados obtidos.

\section{RESULTADOS}

Dos 202 corações estudados, 155 (76.7\%) eram provenientes do sexo masculino e $47(23.3 \%)$ do feminino, prevalecendo a faixa etária entre 56 a 70 anos, com 47 pacientes (31.1\%).

O diagnóstico etiológico da causa base que levou esses pacientes ao transplante cardíaco estava disponível em 51 casos, dos quais $16(31.4 \%)$ eram pacientes com Miocardiopatia Dilatada (MCP), 9 (17.6\%) com MCP-Isquêmica, 7 (13.7\%) com MCPChagásica, 6 (11.8\%) divididos entre MCP-Idiopática (Idio), Hipertrófica (Hiper) e 13 (25.5\%) Aterosclerótica (Ateros), outros diagnósticos em $151(74.7 \%)$ casos.

Dos laudos possíveis de avaliação, de um total de 114 (56,4\%), $68(59,6 \%)$ relatavam que as valvas cardíacas não apresentavam alterações, enquanto 46 (40,4\%) informavam alterações.

O levantamento dos laudos dos corações explantados mostrou alteração valvar morfológica em 41 valvas mitrais (36\%), seguida de 38 casos de valva tricúspide $(33,3 \%), 33$ de valva aórtica $(28,9 \%)$ e $32(28,1 \%)$ de valva pulmonar $(28,1 \%)$. Referente às valvas que não apresentavam alteração, observaram-se 82 casos na valva pulmonar $(71,9 \%), 81$ na valva aórtica $(71,1 \%), 76$ casos na valva tricúspide $(66,7 \%)$ e 73 casos na valva mitral $(64 \%)$.

O cruzamento da variável "alteração valvar" com as variáveis sexo, idade e diagnóstico de base mostrou:

\section{Valva Tricúspide}

Dos 114 laudos, $84(73,7 \%)$ eram do sexo masculino e $30(26,3 \%)$ do sexo feminino, sendo que em $26(68,4 \%)$ casos do sexo masculino prevaleceram as alterações naquela valva. Com relação à variável idade, a faixa etária que prevaleceu foi 46 a 55 anos com 33 (31,4\%) casos; porém, a faixa etária que apresentou mais alterações naquela valva foi 56 a 70 anos de idade, com 12 (32,4\%) dos casos. Quanto ao diagnóstico de base e em qual deles essa valva mais apresentou alteração, obteve-se que o diagnóstico de Miocardiopatia Dilatada foi o que mais prevaleceu, aparecendo em 16 (31,4\%) casos com alteração em oito $(44,4 \%)$ casos. (Tabela 1$)$ 
Tabela 1 - Distribuição referente ao cruzamento da Valva Tricúspide com as variáveis: sexo, faixa etária, diagnóstico de base e presença de alteração valvar, dos corações avaliados, São Paulo, 2008. (N: 114)

\begin{tabular}{|c|c|c|c|c|}
\hline & & $\mathrm{N}(\%)$ & $\begin{array}{c}\text { Sem } \\
\text { Alteração }\end{array}$ & $\begin{array}{c}\text { Com } \\
\text { Alteração }\end{array}$ \\
\hline \multirow{2}{*}{ Sexo } & Masculino & $84(73,7 \%)$ & 58 (76,3\%) & $26(68,4 \%)$ \\
\hline & Feminino & $30(26,3 \%)$ & 18 (23.7\%) & $12(31,6 \%)$ \\
\hline \multirow{5}{*}{$\begin{array}{l}\text { Faixa etária } \\
\text { (anos) }\end{array}$} & 10 a 15 & $10(9,5 \%)$ & $8(11,8 \%)$ & $2(5,4 \%)$ \\
\hline & 16 a 35 & $13(12,4 \%)$ & $7(10,3 \%)$ & $6(16,2 \%)$ \\
\hline & 36 a 45 & 21 (20\%) & $15(22,1 \%)$ & $6(16,2 \%)$ \\
\hline & 46 a 55 & $33(31,4 \%)$ & $22(32,4 \%)$ & $11(29,7 \%)$ \\
\hline & 56 a 70 & $28(26,7 \%)$ & $16(23,5 \%)$ & $12(32,4 \%)$ \\
\hline \multirow{5}{*}{$\begin{array}{l}\text { Diagnóstico de } \\
\text { base }\end{array}$} & Dilatada & $16(31,4 \%)$ & $8(24,2 \%)$ & $8(44,4 \%)$ \\
\hline & Isquêmica & $9(17,6 \%)$ & $6(18,2 \%)$ & $3(16,7 \%)$ \\
\hline & Chagásica & $7(13,7 \%)$ & $6(18,2 \%)$ & $1(5,6 \%)$ \\
\hline & $\begin{array}{l}\text { Idio+ Hiper+ } \\
\text { Ateros }^{\star}\end{array}$ & $6(11,8 \%)$ & $3(9,1 \%)$ & $3(16,7 \%)$ \\
\hline & Outros & $13(25,5 \%)$ & $10(30,3 \%)$ & $3(16,7 \%)$ \\
\hline
\end{tabular}

*Idio: Idiopático, Hiper: Hipertrófica, Ateros: Aterosclerose Teste do Qui-quadrado de Pearson e do Teste de Fisher

\section{Valva Mitral}

Nesse tipo de valva, a população que prevaleceu foi o sexo masculino em $84(73,7 \%)$ casos, onde também foram encontrados os maiores índices de alterações valvar com $28(68,3 \%)$ casos. Quanto à idade, a faixa etária que prevaleceu foi 46 a 55 anos em $33(31,4 \%)$ casos, com uma taxa maior de alteraçã na faixa etária entre 56 a 70 anos de idade, com 14 (35,9\%) casos. Miocardiopatia dilatada (MCP) foi o diagnóstico de base mais prevalente em $16(31,4 \%)$ casos com alteração valvar e em $9(47,4 \%)$ desses casos. (Tabela 2$)$

Tabela 2 - Distribuição referente ao cruzamento da Valva Mitral com as variáveis: sexo, faixa etária, diagnóstico de base e presença alteração valvar, dos corações avaliados, São Paulo, 2008. (N: 114)

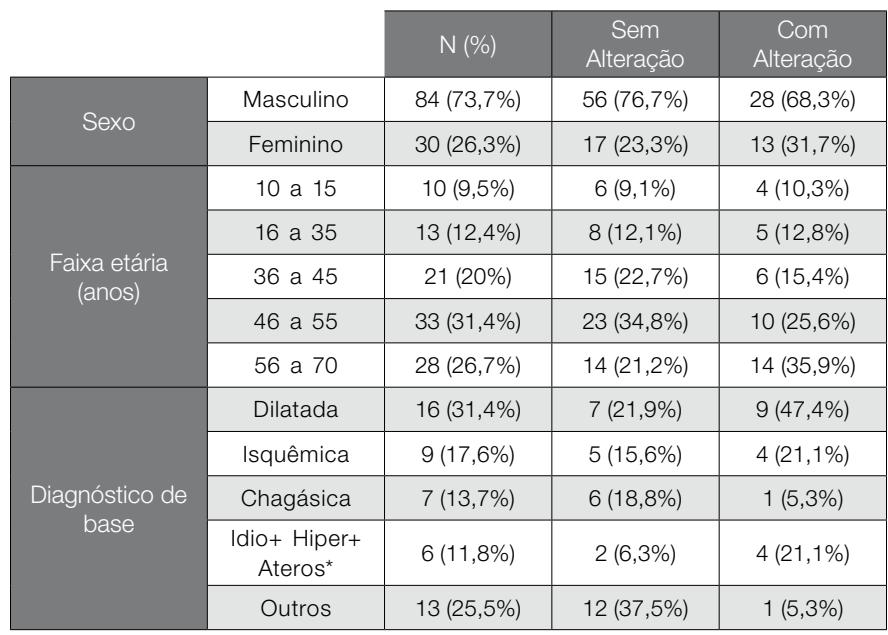

*Idio: Idiopático, Hiper: Hipertrófica, Ateros: Aterosclerose Teste do Qui-quadrado de Pearson e do Teste de Fisher

\section{Valva Pulmonar}

Referente ao sexo, observaram-se 84 casos $(73,7 \%)$ do sexo masculino, prevalecendo também nesse sexo o número maior de alterações, em $22(68,8 \%)$ casos. A faixa etária que mais prevaleceu foi 46 a 55 anos em $33(31,4 \%)$ casos, sendo que a faixa etária entre 56 à 70 anos foi a que mais apresentou alteração, em $11(34,4 \%)$ casos. Miocardiopatia dilatada (MCP) foi mais prevalente como causa, sendo $16(31,4 \%)$ casos, com alteração prevalecendo também nesse diagnóstico em $6(42,9 \%)$ casos. (Tabela 3)

Tabela 3 - Distribuição referente ao cruzamento da Valva Pulmonar com as variáveis: sexo, faixa etária, diagnóstico de base e presença alteração valvar, dos corações avaliados, São Paulo, 2008. (N: 114)

\begin{tabular}{|c|c|c|c|c|}
\hline & & $\mathrm{N}(\%)$ & $\begin{array}{c}\text { Sem } \\
\text { Alteração }\end{array}$ & $\begin{array}{c}\text { Com } \\
\text { Alteração }\end{array}$ \\
\hline \multirow{2}{*}{ Sexo } & Masculino & 84 (73,7\%) & $62(75,6 \%)$ & $22(68,8 \%)$ \\
\hline & Feminino & $30(26,3 \%)$ & $20(24,4 \%)$ & $10(31,3 \%)$ \\
\hline \multirow{5}{*}{$\begin{array}{l}\text { Faixa etária } \\
\text { (anos) }\end{array}$} & 10 a 15 & $10(9,5 \%)$ & $9(12,3 \%)$ & $1(3,1 \%)$ \\
\hline & 16 a 35 & $13(12,4 \%)$ & 7 (9,6\%) & $6(18,8 \%)$ \\
\hline & 36 a 45 & 21 (20\%) & $16(21,9 \%)$ & 5 (15,6\%) \\
\hline & 46 a 55 & $33(31,4 \%)$ & 24 (32,9\%) & $9(28,1 \%)$ \\
\hline & 56 a 70 & $28(26,7 \%)$ & $17(23,3 \%)$ & $11(34,4 \%)$ \\
\hline \multirow{5}{*}{$\begin{array}{c}\text { Diagnóstico de } \\
\text { base }\end{array}$} & Dilatada & $16(31,4 \%)$ & $10(27 \%)$ & $6(42,9 \%)$ \\
\hline & Isquêmica & $9(17,6 \%)$ & $6(16,2 \%)$ & $3(21,4 \%)$ \\
\hline & Chagásica & 7 (13,7\%) & $6(16,2 \%)$ & $1(7,1 \%)$ \\
\hline & $\begin{array}{c}\text { Idio+ Hiper+ } \\
\text { Ateros* }\end{array}$ & $6(11,8 \%)$ & $4(10,8 \%)$ & $2(14,3 \%)$ \\
\hline & Outros & $13(25,5 \%)$ & $11(29,7 \%)$ & $2(14,3 \%)$ \\
\hline
\end{tabular}

*Idio: Idiopático, Hiper: Hipertrófica, Ateros: Aterosclerose Teste do Qui-quadrado de Pearson e do Teste de Fisher

\section{Valva Aórtica}

Prevaleceu sexo masculino, em 84 (73,7\%) casos, e desses, 22 (66,7\%) casos de valvas alteradas. Quanto à idade, a faixa etária foi de 46 a 55 anos em $33(31,4 \%)$ casos, prevalecendo as alterações na faixa etária entre 56 a 70 anos em $11(34,4 \%)$ casos. Sobre o diagnóstico de base, prevaleceu Miocardiopatia Dilatada em $16(31,4 \%)$ casos, e desses, $7(53,8 \%)$ casos com valvas alteradas. (Tabela 4$)$

Tabela 4 - Distribuição referente ao cruzamento da Valva Aórtica com as variáveis: sexo, faixa etária, diagnóstico de base e presença alteração valvar, dos corações avaliados, São Paulo, 2008. (N: 114)

\begin{tabular}{|c|c|c|c|c|}
\hline & & $\mathrm{N}(\%)$ & $\begin{array}{c}\text { Sem } \\
\text { Alteração }\end{array}$ & $\begin{array}{c}\text { Com } \\
\text { Alteração }\end{array}$ \\
\hline \multirow{2}{*}{ Sexo } & Masculino & $84(73,7 \%)$ & $62(76,5 \%)$ & $22(66,7 \%)$ \\
\hline & Feminino & $30(26,3 \%)$ & $19(23,5 \%)$ & $11(33,3 \%)$ \\
\hline \multirow{5}{*}{$\begin{array}{l}\text { Faixa etária } \\
\text { (anos) }\end{array}$} & 10 a 15 & $10(9,5 \%)$ & $7(9,6 \%)$ & $3(9,4 \%)$ \\
\hline & 16 a 35 & $13(12,4 \%)$ & $9(12,3 \%)$ & $4(12,5 \%)$ \\
\hline & 36 a 45 & $21(20 \%)$ & $16(21,9 \%)$ & $5(15,6 \%)$ \\
\hline & 46 a 55 & $33(31,4 \%)$ & 24 (32,9\%) & $9(28,1 \%)$ \\
\hline & 56 a 70 & $28(26,7 \%)$ & $17(23,3 \%)$ & $11(34,4 \%)$ \\
\hline \multirow{5}{*}{$\begin{array}{l}\text { Diagnóstico de } \\
\text { base }\end{array}$} & Dilatada & $16(31,4 \%)$ & $9(23,7 \%)$ & $7(53,8 \%)$ \\
\hline & Isquêmica & $9(17,6 \%)$ & $6(15,8 \%)$ & $3(23,1 \%)$ \\
\hline & Chagásica & $7(13,7 \%)$ & $6(15,8 \%)$ & $1(7,7 \%)$ \\
\hline & $\begin{array}{c}\text { Idio+ Hiper+ } \\
\text { Ateros* }\end{array}$ & $6(11,8 \%)$ & $5(13,2 \%)$ & $1(7,7 \%)$ \\
\hline & Outros & $13(25,5 \%)$ & $12(31,6 \%)$ & $1(7,7 \%)$ \\
\hline
\end{tabular}

*Idio: Idiopático, Hiper: Hipertrófica, Ateros: Aterosclerose Teste do Qui-quadrado de Pearson e do Teste de Fisher 


\section{DISCUSSÃO}

Na casuística apresentada, observou-se que não houve alteração estrutural em $82(71,9 \%)$ casos na valva pulmonar, seguido de 81 $(71,1 \%)$ casos na valva aórtica, $76(66,7 \%)$ na valva tricúspide e 76 $(66,7 \%)$ na valva mitral.

São motivos de exclusão: idade do doador superior a 55 anos, situações clínicas como sepse, neoplasias e doenças auto-imunes, bem como presença de Síndrome de Marfan, demência ou doença neurológica degenerativa. ${ }^{6}$

Em relação à valva aórtica, foram mais frequentes 7 (9,6\%) casos sem alteração na faixa etária compreendida entre 10-15 anos, 9 $(12,3 \%)$ casos entre $16-35$ anos, 16 (21,9\%) casos no intervalo de 36-45 anos e, por fim, 24 (32,9\%) casos entre 46-55 anos, ou seja, passíveis de ser usadas de acordo com critérios estabelecidos por diversos bancos de valvas. ${ }^{8}$

Já com a valva pulmonar, $9(12,3 \%)$ casos não apresentaram alterações morfológicas no intervalo de idade entre 10-15 anos, seguido por $7(9,6 \%)$ casos entre $16-35$ anos, 16 casos $(21,9 \%)$ na faixa etária entre 36-45 anos e 24 (32,9\%) casos entre 46-55 anos, com grande possibilidade de uso. ${ }^{8}$

A experiência do Banco de Valvas Cardíacas Humanas do Hospital de Caridade da Irmandade da Santa Casa de Misericórdia de Curitiba mostra que o uso de valvas aórticas humanas tem sido extremamente significante, com melhor qualidade de vida em relação a pacientes que usam próteses valvares mecânicas e biológicas.

Os dados obtidos sugerem que considerando somente corações explantados de transplantados cardíacos, existe no mínimo a justificativa de captação de valvas para transplante.

\section{CONCLUSÃO}

$\mathrm{O}$ estudo anatomopatológico de corações retirados de receptores cardíacos mostrou a existência de valvas cardíacas que poderiam ser utilizadas em transplante valvular.

Em virtude de dificuldades na obtenção de valvas procedentes de doadores falecidos bem como a escassez de banco de tecidos humanos no Brasil, abre-se a possibilidade com este estudo de pensar na criação de um banco de tecido, em virtude da grande quantidade de valvas que, ao longo dos 16 anos analisados, foram descartadas, pois os enxertos homólogos, sobretudo aórticos, surgem como a melhor alternativa para substituição da valva aórtica em locais com alta incidência de doença cardíaca reumática, como no Brasil.

A crescente demanda por valvas cardíacas humanas (homoenxerto) tem favorecido e justificado a criação do banco de valvas por todo o mundo.

\section{ABSTRACT}

Introduction: Among several heart diseases, the malfunctioning of the human heart valves is outstanding, requiring replacement with biological or metal valves or their replacement by transplantation of human valves coming from deceased donors or living donors as native heart from heart transplant domino donor recipient. Purpose: To investigate the possibility of using the valve tissue from hearts removed in transplantation during the period between 1991 and 2007. Method: This exploratory, descriptive and documentary study was performed in a Public Hospital in Sao Paulo/Brazil, which is reference in cardiovascular disease assistance. Results: The sample consisted of 202 transplanted hearts, prevailing male with 155 (76.7\%) cases, and 47 with age between 56-70 years old (31.1\%). As to the base diagnosis, only $51(25.2 \%)$ had such information, and from these, the most frequent was dilated cardiomyopathy in 16 cases (31.4\%). From 202 hearts assessed, 114 (56.4\%) presented anatomopathological report; from these, 69 (59.6\%) reported that valves had no alteration, while 46 (40.4\%) informed the presence of altered valves. Heart valves with less frequency of alterations were: pulmonary in 82 cases (71.9\%) and aortic valves in 81 cases (71.1\%). Conclusion: The anatomopathological study of hearts collected from heart receptors showed the existence of heart valves which could be used in valve transplants, mainly aortic valve.

Keywords: Heart valves; Heart Valve Prosthesis Implantation; Tissue Donors; Heart Transplantation; Tissue transplantation.

\section{REFERÊNCIAS}

1. Camargo et al. Doação de Órgãos - Uma responsabilidade de todos. Vol. 5, pg. 1095, 1994.

2. Pereira WA. et al. Manual de Transplante de Órgãos e Tecidos; Rio de Janeiro, Editora Guanabara-Koogan, $3^{\circ}$ Edição, 2004.

3. Filho AP; Martins MP; Andrade, VS. Transplante Cardíaco. [Monografia]. São Paulo, 2002 .

4. Bacal F, Souza-Neto JD, Fiorelli AI, Mejia J, Marcondes-Braga FG, Mangini S, et al. II Diretriz Brasileira de Transplante Cardíaco. Arq Bras Cardiol. 2009;94(1 supl.1):e16-e73

5. Luckraz H, Sharples L, Charman S, Tsui S, Wallwork J, Parameshwar J, et al Does heart transplantation confer survival benefit in all risk groups? J Heart Lung Transplant. 2005; 24 (9):1231-4.

6. O’Brrien MF, Harrocks S, Stafford EG, Gardner MA, Pohlner PG, Tesar PJ et al
The homograft aortic valve: a 29 year, $99.3 \%$ follow up of 1,022 valve replacements. J Hearth Valve Dis. 2001;10(3):334-45.

7. Ross, DN. Aortic root replacement with a pulmonaty autograft - Current trends. J Heart Valve Dis. 1994;3:358-60.

8. Costa, MTBA; Costa, FDA; Nazareno, LCF; Domachoski, J; Peruzzo, AM; Colatusso, C. et al. IA. Análise das atividades dos oito anos iniciais do Banco de Valvas Cardíacas Humanas do Hospital de Caridade da Irmandade da Santa Casa de Misericórdia de Curitiba. Braz J Cardiovasc Surg. 2005;20(4):398-407.

9. Verghese, S; Padmaja, P; Sindhu, B; Elizabeth, SJ; Lesley, N; Cherian, KM. Homograft Valve Bank: Our Experience in Valve Banking. Indian Heart J. 2004;56:299-306.

10. Lei 9.434 de 04 de Fevereiro de 1997. Dispõe sobre a remoção dos órgãos, tecidos e partes do corpo humano para fins de transplante e tratamento e dá outras providências. 